Check for updates

Cite this: J. Mater. Chem. C, 2022, 10, 4851

Received 18th October 2021, Accepted 21st December 2021

DOI: $10.1039 / \mathrm{d} 1 \mathrm{tc} 05026 \mathrm{e}$

rsc.li/materials-c

\title{
Enhancement of thermally activated delayed fluorescence properties by substitution of ancillary halogen in a multiple resonance-like diplatinum(II) complex†
}

\author{
Piotr Pander, (D)*ab Andrey V. Zaytsev, (D) ${ }^{c}$ Amit Sil, (D) d J. A. Gareth Williams, (D)*d \\ Valery N. Kozhevnikov (D) *c and Fernando B. Dias (D) ${ }^{\text {a }}$
}

\begin{abstract}
We present an in-depth investigation of the influence of chloro-to-iodo exchange on the thermally activated delayed fluorescence (TADF) of a dinuclear platinum(॥) complex featuring monodentate halide ancillary ligands. The complexes are constructed using a ditopic bis $-\mathrm{N}^{\wedge} \mathrm{C}^{\wedge} \mathrm{N}$-chelating ligand of the form $\left(\mathrm{N}^{\wedge} \mathrm{C}^{\wedge} \mathrm{N}-\mathrm{N}^{\wedge} \mathrm{C}^{\wedge} \mathrm{N}\right) \mathrm{Pt}_{2} \mathrm{X}_{2}$. The initially formed chloro complex $(X=\mathrm{Cl})$ is readily transformed into the iodo analogue $(X=1)$. The change is found to increase the radiative decay rate constant $k_{r}$ by around 3 fold to $3-4 \times 10^{5} \mathrm{~s}^{-1}$. This remarkably high value is comparable with the state-of-the-art iridium(III) organometallic phosphors. The improved luminescence properties of the iodo compound are shown to be due to a smaller singlet-triplet energy gap $\Delta E_{\mathrm{ST}}$ compared to the chloro analogue. lodide reduces the HOMO-LUMO overlap in a multiple resonance-like orbital structure of the complex. lodination is therefore the first practical strategy shown to improve the TADF properties of diplatinum(॥) complexes. The analogous monoplatinum(॥) phosphorescent complex is studied in parallel in order to shed light more generally on the effect of iodo ligands on the triplet and singlet states and on spin-orbit coupling (SOC) in platinum(॥) complexes.
\end{abstract}

\section{Introduction}

Highly luminescent iridium(III) and platinum(II) organometallic compounds have become widespread in OLEDs in recent years due to their high stability and short phosphorescence lifetimes. ${ }^{1-5}$ The triplet radiative rate constants $\left(k_{\mathrm{r}}^{\mathrm{T}}\right)$ of such complexes are generally four to five orders of magnitude higher than those of metal-free organic phosphors. ${ }^{6}$ Efforts towards further accelerating their radiative decay, to reduce the influence of luminescence quenching processes in devices, have

\footnotetext{
${ }^{a}$ Department of Physics, Durham University, South Road, Durham, DH1 3LE, UK. E-mail: piotr.h.pander@durham.ac.uk

${ }^{b}$ Centre for Organic and Nanohybrid Electronics, Silesian University of Technology, Konarskiego 22B, 44-100 Gliwice, Poland

'Department of Applied Sciences, Northumbria University, Ellison Building, Newcastle upon Tyne, NE1 8ST, UK.

E-mail: valery.kozhevnikov@northumbria.ac.uk

${ }^{d}$ Department of Chemistry, Durham University, South Road, Durham, DH1 3LE,

UK. E-mail: j.a.g.williams@durham.ac.uk

$\dagger$ Electronic supplementary information (ESI) available: Synthetic details and characterisation of new materials; X-ray diffraction and crystal data; further information on the equipment and methods for theory, photophysical characterisation, electrochemistry, and OLED devices. Crystallographic data for complex 3. CCDC 2121802. For ESI and crystallographic data in CIF or other electronic format see DOI: 10.1039/d1tc05026e
}

mostly focused on strategies that increase the metal character of the excited states to enhance spin-orbit coupling (SOC). ${ }^{1,7}$ Recently, it has been found that the use of dinuclear structures - featuring two metal ions - may lead to larger SOC and accelerated triplet radiative rate constants relative to structurally comparable mononuclear complexes. ${ }^{8-10}$ Meanwhile, the decay rates of organometallic complexes may also be increased if they exhibit thermally activated delayed fluorescence (TADF). ${ }^{11,12}$ This phenomenon relies on the up-conversion of triplets into singlets $\left(\mathrm{T}_{1} \rightarrow \mathrm{S}_{1}\right)$ and thus benefits from the high singlet radiative rates $\left(S_{1} \rightarrow S_{0}\right)$ rather than relying on the promotion of the triplet radiative rate constant $\left(\mathrm{T}_{1} \rightarrow \mathrm{S}_{0}\right) .{ }^{1}$ It results in radiative decay rates being less dependent on SOC than those of conventional phosphorescent complexes. TADF shows particular promise for promoting $k_{\mathrm{r}}$ in red and near infrared (NIR) organometallic emitters, where SOC is invariably diminished due to increased ligand character to the excited state upon extending the ligand conjugation, for example. ${ }^{13,14}$

So far, TADF properties have been found in a plethora of metal-free compounds ${ }^{15,16}$ as well as in organometallic complexes of $\mathrm{Cu}(\mathrm{I}),{ }^{17-19} \mathrm{Ag}(\mathrm{I}),{ }^{20} \mathrm{Au}(\mathrm{I})^{21}$ and $\mathrm{Pd}(\mathrm{II}),{ }^{11,22,23}$ but lesser known examples involving other metal ions such as $\mathrm{W}(\mathrm{vI})^{24}$ or $\mathrm{Sn}(\mathrm{Iv})^{25}$ also exist. The latest discovery of TADF properties in dinuclear platinum(II) ${ }^{26}$ and of somewhat similar behaviour in 

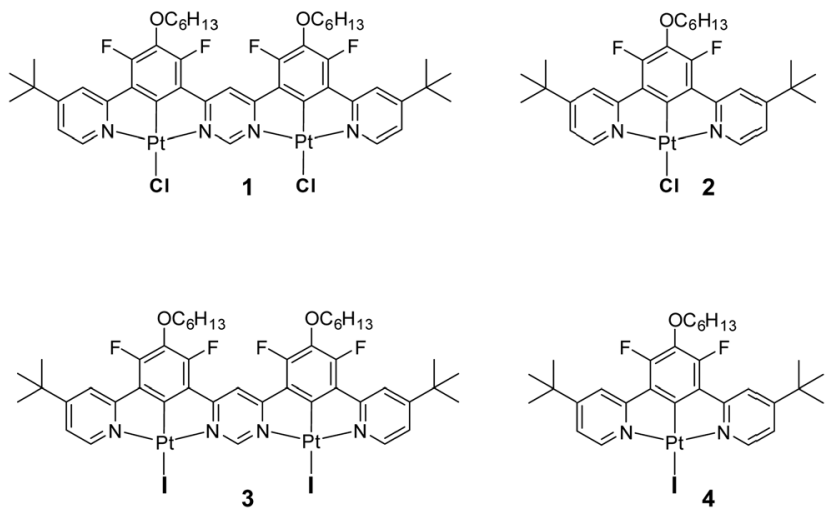

Chart 1 Top: Structure of the dinuclear Pt(II) complex 1 recently discovered to show $\operatorname{TADF}^{28}$ and of its mononuclear analogue 2 , which feature monodentate chloro ligands. Bottom: The structures of the iodo derivatives $\mathbf{3}$ and $\mathbf{4}$ prepared and studied in the current work, being the analogues of 1 and 2 respectively.

di- and mononuclear iridium(III) ${ }^{27}$ complexes has demonstrated that large radiative rates can be obtained without the need for strong SOC from the metal. Further research has indicated that dinuclear complexes of platinum(II) are more likely to demonstrate TADF than their mononuclear counterparts, as the former have smaller $\Delta E_{\mathrm{ST}}$ and larger $\mathrm{S}_{1} \rightarrow \mathrm{S}_{0}$ radiative rates - factors crucial for efficient delayed fluorescence. ${ }^{28}$ There persists a need, however, to better understand all the effects that lead to efficient TADF in this newly emerged group of emitters. Having recently studied the first example of a $\mathrm{Pt}(\mathrm{II})$ complex 1 involving a ditopic bis- $\mathrm{N}^{\wedge} \mathrm{C}^{\wedge} \mathrm{N}$ chelating ligand that demonstrates TADF under ambient conditions, we sought possible ways to reduce $\Delta E_{\mathrm{ST}}$ and thus further promote the radiative decay. In this contribution, we show how such an effect can be readily achieved through metathesis of the halide co-ligands from chloro to iodo (compound 3) and we also probe the effect of iodo ligands more generally by studying the mononuclear complex 4 (Chart 1).

\section{Synthesis}

The rationale behind the use of ditopic, bis- $\mathrm{N}^{\wedge} \mathrm{C}^{\wedge} \mathrm{N}$ coordinating ligands has been discussed in our earlier work. ${ }^{28}$ Briefly, $\mathrm{N}^{\wedge} \mathrm{C}^{\wedge} \mathrm{N}$-coordinating ligands - especially those based on 2,6-dipyridylbenzenes - have been shown to form cyclometallated complexes with metal ions such as $\mathrm{Pt}(\mathrm{II})($ e.g., 2) and $\operatorname{Ir}(\mathrm{III}){ }^{27}$ The short $\mathrm{M}-\mathrm{C}$ bonds coupled with the strong $\sigma$ donation properties of the metallated ring ensure signficant metal character to the lowest-energy excited states, and hence efficient spin-orbit coupling. ${ }^{29}$ The synergy between such $\sigma$ donation and $\pi^{*}$ acceptor character of the heterocycles meanwhile ensures that potentially deactivating $\mathrm{d}-\mathrm{d}$ states are kept high in energy. Finally, the rigidity associated with such structures helps to minimise non-radiative decay pathways. ${ }^{30}$ The coupling of two such $\mathrm{N}^{\wedge} \mathrm{C}^{\wedge} \mathrm{N}$ units together through a mutually common pyrimidine ring offers an attractive route to dinuclear complexes where these desirable features are not only retained, but are also accompanied by significant lowering of the emissive excited state energy and enhancement of the radiative rate constant. ${ }^{31}$

The synthesis of the derivative incorporating fluoro, hexoxy, and tert-butyl substituents (enhancing solubility of the ensuing complex) has been described in our earlier work, together with its metallation with two $\mathrm{Pt}(\mathrm{II})$ ions. In the present work, the resulting dinuclear complex $\mathbf{1}$ featuring monodentate chloro ligands has been transformed to the diiodo derivative $\mathbf{3}$ upon treatment with an excess of sodium iodide in acetone under reflux (see Section S2, ESI $\dagger$ ). The mononuclear model 4 was similarly obtained from 2 . The identity and purity of the two new complexes were confirmed by ${ }^{1} \mathrm{H},{ }^{13} \mathrm{C},{ }^{19} \mathrm{~F}$ NMR spectroscopy and by elemental analysis. Crystals of 3 suitable for X-ray diffraction analysis were obtained from several separate crystallisations. Although the data could not be refined to a level sufficient for in-depth consideration of bond lengths and angles, the identity of the complex is unequivocally confirmed (see Section S3, ESI $\dagger$ ).

\section{Results and discussion}

\section{Solution state photophysics}

Steady-state absorption and emission spectra. The absorption and photoluminescence spectra of $\mathbf{3}$ and $\mathbf{4}$ in dilute solutions are shown in Fig. 1 while the photoluminescence properties of both complexes are summarised in Table 1. It immediately becomes apparent that the photoluminescence of dinuclear complex $\mathbf{3}$ is red-shifted compared to $\mathbf{4}$, which is due to the larger $\pi$-conjugated system in the former. Both complexes demonstrate very weak effects of solvent polarity on their photoluminescence (Fig. 2), which indicates that the emissive excited states do not involve net shifts in electron density with respect to $S_{0}$.

Most strikingly however, the photoluminescence and absorption spectra of 3 clearly overlap, indicating that the emissive state might be associated with a radiative transition possessing large oscillator strength $\left(\varepsilon \sim 10^{4} \mathrm{M}^{-1} \mathrm{~cm}^{-1}\right)$, such as of singlet and not triplet character. ${ }^{11,23,26,28}$ On the other hand, 4 presents a typical picture of a phosphorescent transition metal complex with a relatively large apparent Stokes shift and no overlap of the emission spectrum with transitions of high oscillator strength. As this behaviour of complex 3 is in

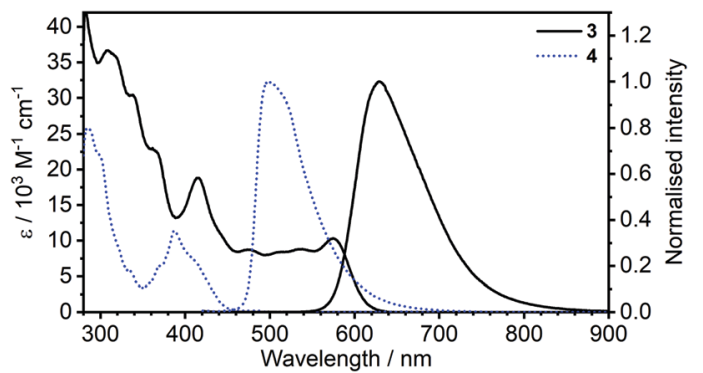

Fig. 1 Comparison of absorption and photoluminescence spectra of complexes 3 and 4 in toluene, $c=10^{-5} \mathrm{M}$ for absorption and $\mathrm{c}=$ $5 \times 10^{-7} \mathrm{M}$ for emission spectra. 
Table 1 Summary of spectroscopic properties of $\mathbf{3}$ and $\mathbf{4}$ in degassed solvents at room temperature

\begin{tabular}{|c|c|c|c|c|c|c|c|}
\hline Complex & Solvent & $\lambda_{\mathrm{abs}}{ }^{a} / \mathrm{nm}\left(\varepsilon / \mathbf{M}^{-1} \mathrm{~cm}^{-1}\right)$ & $\begin{array}{l}\lambda_{\mathrm{em}}{ }^{b} / \\
\mathrm{nm}\end{array}$ & $\Phi_{\mathrm{PL}}{ }^{c}$ & $\begin{array}{l}\tau^{d} / \\
\mu \mathrm{s}\end{array}$ & $\begin{array}{l}k_{\mathrm{r}}^{e} / \\
10^{5} \mathrm{~s}^{-1}\end{array}$ & $\begin{array}{l}k_{\mathrm{nr}}^{f} / \\
10^{5} \mathrm{~s}^{-1}\end{array}$ \\
\hline \multirow[t]{3}{*}{3} & Toluene & $\begin{array}{l}575 \text { (10 300), } 536 \text { (8800), } 509(8400), 475(8700), 443 \text { (10 800), } 415 \text { (18 800), 368sh (22 300), } \\
339 \text { (30 400), 318sh (35 800), } 309(36700)\end{array}$ & 628 & 0.43 & 1.0 & 4.3 & 5.6 \\
\hline & Chlorobenzene & $\begin{array}{l}571(10100), 538(8200), 511 \text { (7900), 473sh (7500), } 411 \text { (16 800), 363sh (23 400), } 337 \text { (27 000), } \\
311(31300)\end{array}$ & 633 & 0.57 & 1.7 & 3.3 & 2.5 \\
\hline & $\mathrm{CH}_{2} \mathrm{Cl}_{2}$ & $\begin{array}{l}555 \text { (9800), } 528 \text { (8700), } 503 \text { (8200), } 463 \text { (7300), 439sh (8700), } 402 \text { (16 500), 374sh (22 000), } 353 \\
(26400), 332 \text { (27 800), } 309 \text { (32 900) }\end{array}$ & 635 & 0.27 & 1.5 & 1.8 & 5.0 \\
\hline \multirow[t]{3}{*}{4} & Toluene & $\begin{array}{l}478 \text { (200), 411sh (7300), } 388(11400), 370 \text { sh (6400), 336sh (5700), 300sh (22 000), } 285 \\
(25700)\end{array}$ & 498 & 0.82 & 5.1 & 1.6 & 0.4 \\
\hline & Chlorobenzene & 479 (200), 412sh (5900), $387(10600), 368 \mathrm{sh}(6000), 336$ (5700), 299sh (21 500) & 510 & 0.83 & 5.6 & 1.5 & 0.3 \\
\hline & $\mathrm{CH}_{2} \mathrm{Cl}_{2}$ & 475 (200), 404sh (6300), 381 (11300), 361sh (7000), 333 (7500), 288 (29600), 259 (35 100) & 511 & 0.83 & 6.0 & 1.4 & 0.3 \\
\hline
\end{tabular}

${ }^{a}$ Absorption maxima and molar absorption coefficients. ${ }^{b}$ Emission maxima. ${ }^{c}$ Photoluminescence quantum yield recorded against rhodamine $6 \mathrm{G}$ $\left(\Phi_{\mathrm{PL}}=0.91^{33}\right)$ or coumarin $153\left(\Phi_{\mathrm{PL}}=0.53^{33}\right)$ in air-equilibrated absolute ethanol solutions. ${ }^{d}$ Photoluminescence lifetime at room temperature. ${ }^{e}$ Observed radiative rate constant, $k_{\mathrm{r}}=\Phi_{\mathrm{PL}} / \tau$. ${ }^{f}$ Observed non-radiative rate constant, $k_{\mathrm{nr}}=\left(1-\Phi_{\mathrm{PL}}\right) / \tau$.
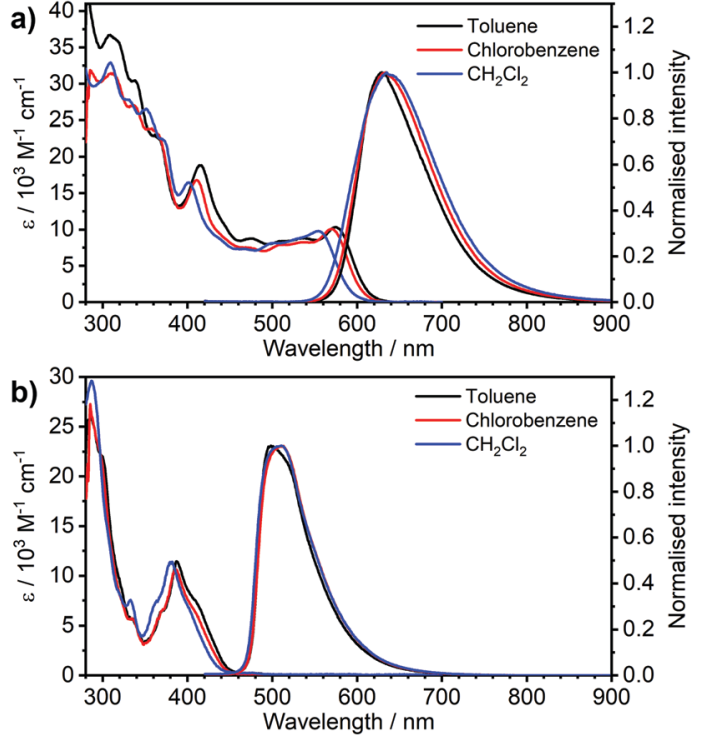

Fig. 2 Absorption and photoluminescence spectra of complexes (a) 3 and (b) 4 in the three solvents indicated in the figure legends, $c \approx 10^{-5} \mathrm{M}$ for absorption and $c=5 \times 10^{-7} \mathrm{M}$ for emission spectra.

common with other recently discovered diplatinum(II) complexes $^{26,28}$ that demonstrate TADF properties, we have studied the temperature-dependent behaviour of its photoluminescence in toluene solution (Fig. 3 and 4). The photoluminescence intensity of 3 increases by $\sim 25 \%$ upon decreasing the temperature from 300 to $230 \mathrm{~K}$, which is attributed to the suppression of non-radiative decay pathways affecting the triplet state. At temperatures below $230 \mathrm{~K}$ we note a strong decline in photoluminescence intensity and an associated change in the spectrum, which becomes more clearly visible at temperatures close to $160 \mathrm{~K}$. Further analysis allows the emission to be deconvoluted into the sum of two separate emission spectra: energetically higher, attributed to TADF $\left(\lambda_{\mathrm{em}}=627 \mathrm{~nm}\right)$, and lower, attributed to phosphorescence $\left(\lambda_{\mathrm{em}}=671 \mathrm{~nm}\right)$ (Fig. S5.4, ESI $\left.\dagger\right)$. Having made this analysis we observe a significant decline in the TADF intensity and a small

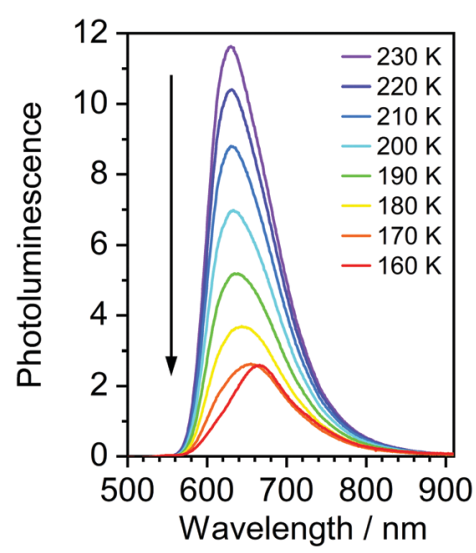

Fig. 3 Photoluminescence spectra of 3 in toluene at various temperatures from 230 to $160 \mathrm{~K}, \mathrm{c}=10^{-5} \mathrm{M}$.

associated increase in phosphorescence intensity upon cooling from $230 \mathrm{~K}$ to $160 \mathrm{~K}$. The ratio of TADF to phosphorescence shows a trend consistent with the models used in early studies of the phenomenon ${ }^{32}$ which allows the activation energy for the process to be estimated as $E_{\mathrm{a}}=116 \pm 2 \mathrm{meV}$. We also note the TADF-to-phosphorescence ratio does not follow the trend in the temperature range above $230 \mathrm{~K}$ - where non-radiative processes strongly affect the $\mathrm{T}_{1}$ state. Although the photoluminescence spectrum of 3 at $300 \mathrm{~K}$ is clearly dominated by TADF, phosphorescence still contributes to $\sim 7 \%$ of the total photoluminescence intensity (Fig. S5.4, ESI $\dagger$ ).

Time-resolved photoluminescence. Photoluminescence decay properties of $\mathbf{3}$ and $\mathbf{4}$ at room temperature are summarised in Table 1 . The photoluminescence lifetime and quantum yield $\Phi_{\mathrm{PL}}$ of $\mathbf{4}$ do not show any significant variation with solvent polarity, and the radiative rate constant remains at $k_{\mathrm{r}}=1.4-1.6 \times 10^{5} \mathrm{~s}^{-1}$ in all three solvents used in this study. On the other hand, 3 shows a significant variation of $\Phi_{\mathrm{PL}}$ and $k_{\mathrm{r}}$ with solvent polarity, despite the solvatochromism in photoluminescence being insignificant, with only a $7 \mathrm{~nm}$ shift in $\lambda_{\mathrm{em}}$ between toluene and $\mathrm{CH}_{2} \mathrm{Cl}_{2}$. This can be attributed to a fine tuning of the $\Delta E_{\mathrm{ST}}$ due to weak solvatochromic shifts in $\mathrm{S}_{1}$ and 


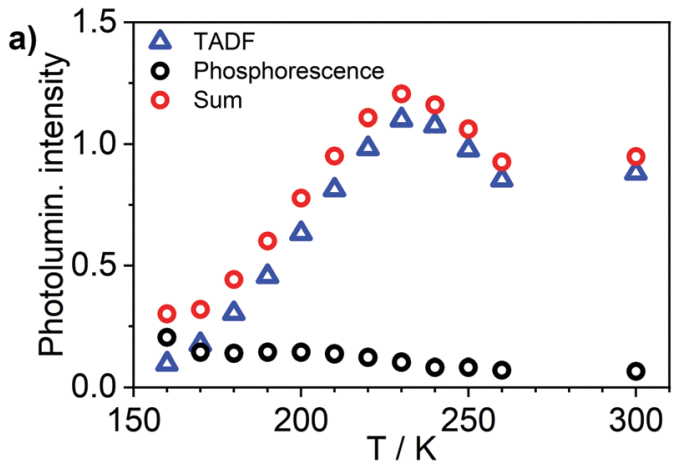

b)

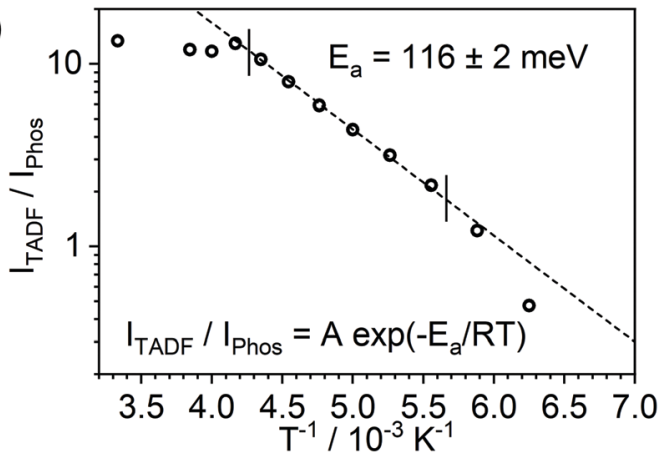

Fig. 4 Photoluminescence of complex 3 in toluene solution $\left(c=10^{-5} \mathrm{M}\right)$ as a function of temperature: (a) intensity of TADF, intensity of phosphorescence and their sum (total intensity); (b) ratio of TADF to phosphorescence.

$\mathrm{T}_{1}$ energy (calculated dipole moments for complexes $\mathbf{3}$ and $\mathbf{4}$ are presented in Table S4.11, ESI $\dagger$ ). The shortest photoluminescence lifetime and largest decay rate constant were observed in toluene, hence the selection of this solvent for the temperaturedependent studies of the TADF complex 3 . We note that, in toluene, the $k_{\mathrm{r}}$ of 3 is up to 3 -fold larger than that of 4 , which is the consequence of the TADF mechanism being at work in the former. The divergent behaviour of $\mathbf{3}$ and $\mathbf{4}$ agrees with earlier findings which suggest diplatinum(II) complexes are more likely to show delayed fluorescent behaviour. ${ }^{28}$

The photoluminescence decay of $\mathbf{3}$ was studied as a function of temperature in toluene (Fig. 5). At each temperature, the decay lifetime recorded at $630 \mathrm{~nm}$ is identical with the values registered at 650 and $700 \mathrm{~nm}$ (Fig. S5.6 and S5.7, ESI $\dagger$ ) (note phosphorescence maximum at $\lambda_{\mathrm{em}}=671 \mathrm{~nm}$ ), which suggests that the two emissive states involved in the luminescent process, $\mathrm{S}_{1}$ and $\mathrm{T}_{1}$, remain in equilibrium. ${ }^{1,34}$ This behaviour of the photoluminescence decay, ${ }^{34}$ together with the other experimental features presented in this work, provide an unequivocal confirmation for the TADF mechanism being at work. The dependence of the observed lifetime with temperature is well fitted using the model described by Eqn $1^{1,35}$ in Fig. 5, Fig. S5.6 and S5.7 (ESI $\dagger$ ), where $\tau_{\text {obs }}(T)$ is the observed emission lifetime (s); $E_{\mathrm{a}}$ is the activation energy of the reverse intersystem crossing process in $\mathrm{J} \mathrm{mol}^{-1} ; \tau_{\mathrm{PH}}$ is the phosphorescence lifetime $(\mathrm{s}) ; k_{\mathrm{r}}^{\mathrm{S}}$ is the radiative rate constant of the singlet state $\left(\mathrm{s}^{-1}\right) ; R$ is the universal gas constant,

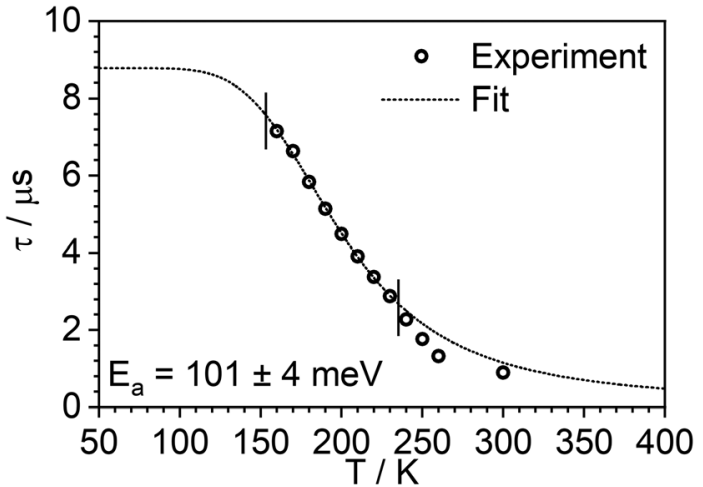

Fig. 5 Photoluminescence decay lifetime as a function of temperature in 3 at $635 \mathrm{~nm}$ and the best fit according to Eqn (1). Note that experimental data points at higher temperature are excluded from the fit, as they are found from the steady-state experiments to be affected by changes in non-radiative decay

$8.314 \mathrm{~J} \mathrm{~mol}^{-1} \mathrm{~K}^{-1}$; and $T$ is the temperature in $\mathrm{K}$. The model yields $E_{\mathrm{a}}=101 \pm 4 \mathrm{meV}$ which is close to the value obtained from the energy difference between $S_{1}$ and $T_{1}$ measured from the onset of the deconvoluted steady state emission spectra.

$$
\tau_{\text {obs }}(T)=\frac{3+\mathrm{e}^{-\frac{E_{\mathrm{a}}}{R T}}}{\frac{3}{\tau_{\mathrm{PH}}}+k_{\mathrm{r}}^{\mathrm{S}} \mathrm{e}^{-\frac{E_{\mathrm{a}}}{R T}}}
$$

With the experimental picture presented by complex 3, the energetically higher state is associated with a transition demonstrating faster radiative decay to the ground state than the lower-energy luminescent state. Thus the former is attributed to a singlet and the latter to a triplet state. Such attribution of the emissive state multiplicity justifies the use of the term delayed fluorescence in the description of the experimental findings.

\section{Solid state photophysics}

Solid films are a convenient medium for studying luminescent complexes at cryogenic temperatures as they do not undergo phase transitions (freezing/melting) in the 300-80 K temperature window. Therefore, to further confirm the TADF properties of complex 3, we have studied the photoluminescence decay, as well as the steady-state and time-resolved photoluminescence spectra as a function of temperature in polystyrene films (Fig. 6 and Fig. S5.9-S5.12, ESI $\dagger$ ). A similar analysis was made for the phosphorescent complex 4 and is presented in the ESI $\dagger$ (Fig. S5.13 and S5.14).

The photoluminescence behaviour of 3 in polystyrene matrix is in agreement with that in toluene solution. In this case, however, the $E_{\mathrm{a}}=58 \pm 3 \mathrm{meV}$ (obtained by fitting photoluminescence lifetime as a function of temperature with Eqn (1)) is significantly smaller than in toluene. This also leads to the phosphorescence and TADF having very similar spectra so that the change in contribution of both components observed in function of temperature is less striking than in toluene 
a)
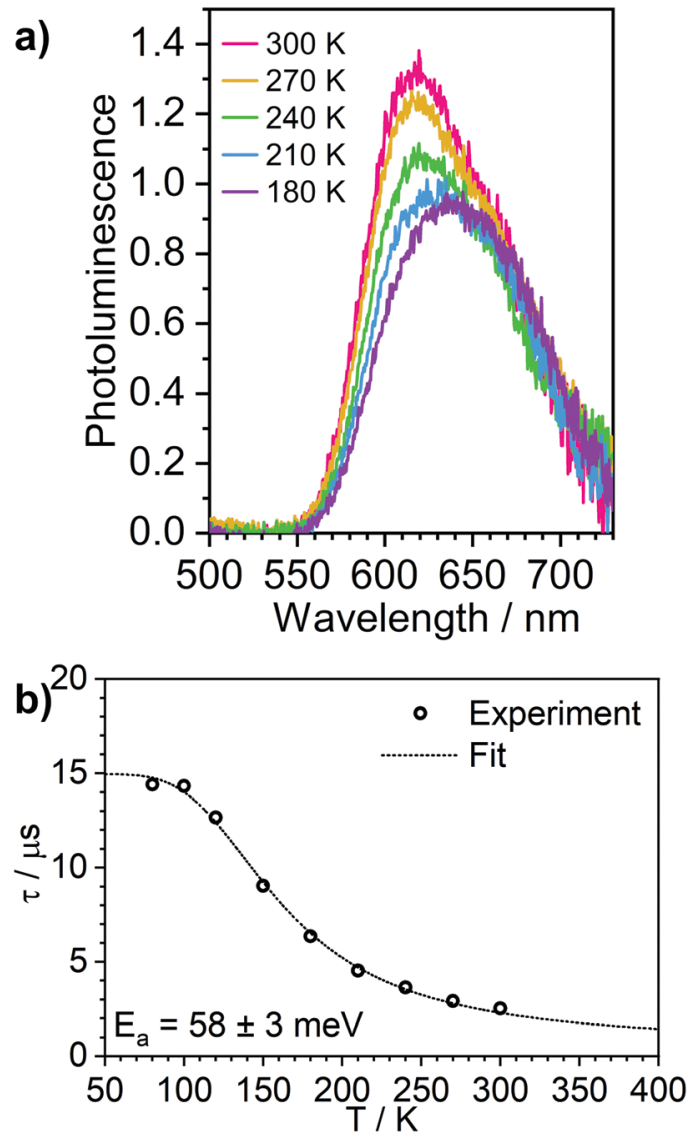

Fig. 6 Photoluminescence of 3 in polystyrene matrix at $0.1 \%(\mathrm{w} / \mathrm{w})$. (a) steady state spectra recorded at temperatures from 300 to $180 \mathrm{~K}$. (b) photoluminescence decay lifetime at temperatures from 300 to $80 \mathrm{~K}$, showing the experimental data points and the best fit according to Eqn (1)

solution. The radiative rate of the singlet state, $k_{\mathrm{r}}^{\mathrm{S}}=1 \times 10^{7} \mathrm{~s}^{-1}$, obtained from the fit is nearly an order of magnitude smaller than that reported in $\mathbf{1} .^{28}$
Explaining the role of the ancillary halogen ligand using DFT and TDDFT calculations

Density functional theory (DFT) and time-dependent DFT (TDDFT) calculations at the B3LYP/def2-TZVP/ZORA level of theory were employed in order to explain the divergent behaviour between complexes $\mathbf{1}$ and 2, and their iodo-analogues 3 and 4. First of all, we recognise that iodine, being a heavier halogen than chlorine, should introduce additional SOC to the complex. It is widely recognised that heavier halogens, $\mathrm{Br}$ and I, in metal-free organic molecules are often implicated in the room temperature phosphorescence (RTP) properties of such compounds and numerous examples are known. ${ }^{36-41}$ Heavy halogens, however, induce relatively long-lived RTP in comparison with typical platinum(II) or iridium(III) organometallic compounds, which can be attributed to differences in electron structure favouring the mixing of $\mathrm{p}$ orbitals into the excited state in halogens rather than $d$ orbitals as with transition metals. Interestingly, efficient emission from a di-iridium(III) complex has been interpreted recently in terms of the strong SOC of $\mathrm{T}_{1}$ with singlets, associated with the change from chloride to iodide co-ligands. ${ }^{42}$ Such an effect is favoured by increasing halide to heterocycle charge-transfer character, as observed previously for $\operatorname{Re}\left(\mathrm{N}^{\wedge} \mathrm{N}\right) \mathrm{CO}_{3} \mathrm{X}$ complexes (where $\mathrm{X}=$ halide), for example. ${ }^{43,44}$

We first simulate the electronic properties of the phosphorescent monoplatinum(II) analogues $\mathbf{2}$ and $\mathbf{4}$ as a control group for the TADF complexes 1 and 3. Given the good correlation between simulated and experimental properties obtained earlier ${ }^{28}$ for $\mathbf{1}$ and $\mathbf{3}$, we again follow the approach of Younker and Dobbs ${ }^{45}$ to study molecules at their ground state geometry. The analysis of excited states is performed at two levels of theory: routine time-dependent density functional theory (TDDFT) and TDDFT including spin-orbit coupling (SOCTDDFT). We will refer to the former as TDDFT or zero-order states $\left(\mathrm{S}_{n}, \mathrm{~T}_{n}\right)$, and to the latter as SOC-TDDFT states $\left(\Gamma_{n}\right)$, where

Table 2 Comparison of experimental luminescent properties of $\mathrm{Pt}-\mathrm{N}^{\wedge} \mathrm{C}^{\wedge} \mathrm{N}-\mathrm{X}$ complexes $\mathbf{3}$ and $\mathbf{4}$ and their analogues $\mathbf{1}$ and $\mathbf{2}$. Experimental values for $\mathbf{1}$ and 2 were reported in earlier work ${ }^{28}$

\begin{tabular}{|c|c|c|c|c|c|}
\hline & $\lambda_{\mathrm{em}}^{b} / \mathrm{nm}$ & 578 sh, 635 & 633 & 514 & 510 \\
\hline & $\tau^{d} / \mu \mathrm{s}$ & 5.0 & 1.7 & 5.7 & 5.6 \\
\hline & $k_{\mathrm{r}}^{e} / 10^{5} \mathrm{~s}^{-1}$ & 1.0 & 3.3 & 1.5 & 1.5 \\
\hline & $k_{\mathrm{nr}} f_{h} / 10^{5} \mathrm{~s}^{-1}$ & 1.0 & 2.5 & 0.3 & 0.3 \\
\hline \multirow[t]{6}{*}{ Polystyrene } & $\lambda_{\mathrm{em}}^{b} / \mathrm{nm}$ & 579sh, 640 & 618 & 498 & 502 \\
\hline & $k_{\mathrm{r}}^{\mathrm{S} h} / \mathrm{s}$ & $9 \times 10^{7}$ & $1 \times 10^{7}$ & - & - \\
\hline & $E_{\mathrm{a}}{ }^{i} / \mathrm{eV}$ & $0.159 \pm 0.020$ & $0.058 \pm 0.003$ & - & - \\
\hline & $\mathrm{S}_{1}^{j} / \mathrm{eV}$ & 2.30 & 2.20 & - & - \\
\hline & $\mathrm{T}_{1}{ }^{k} / \mathrm{eV}(80 \mathrm{~K})$ & 2.10 & 2.14 & 2.68 & 2.67 \\
\hline & $\Delta E_{\mathrm{ST}}^{l} / \mathrm{eV}$ & 0.20 & 0.06 & - & - \\
\hline
\end{tabular}

${ }^{a}$ Absorption spectrum onset. ${ }^{b}$ Emission maxima. ${ }^{c}$ Photoluminescence quantum yield. ${ }^{d}$ Photoluminescence lifetime at room temperature. ${ }^{e}$ Observed radiative rate constant, $k_{\mathrm{r}}=\Phi_{\mathrm{PL}} / \tau$. ${ }^{f}$ Observed non-radiative rate constant, $k_{\mathrm{nr}}=\left(1-\Phi_{\mathrm{PL}}\right) / \tau$. ${ }^{g}$ Photoluminescence lifetime at $80 \mathrm{~K}$. ${ }^{h}$ Singlet radiative decay rate. ${ }^{i}$ Activation energy of the TADF process. ${ }^{j}$ Singlet energy recorded from fluorescence (TADF) spectrum onset at room temperature. ${ }^{k}$ Triplet energy recorded from phosphorescence spectrum onset at $80 \mathrm{~K} .{ }^{l}$ Singlet-triplet energy splitting. Figures for complexes 1 and 2 are reproduced from earlier work. ${ }^{28}$ Some of the values for complexes 3 and 4 are reproduced from Table 1. 
$\Gamma_{0}$ denotes ground state. The single point energy calculations of TDDFT and SOC-TDDFT states have been performed using the ground state $\left(\mathrm{S}_{0}\right)$ geometry optimised at the B3LYP/def2-TZVP level of theory. Further details are given in Section 1. General of the ESI. $\dagger$

We have not observed any significant differences in luminescent properties between 2 and 4 (Table 2), nor in the calculations (Table S4.1, ESI $\dagger$ ). We note however that complex 4 displays slightly lower experimental energy gap than $\mathbf{3}$, while the triplet energy from the phosphorescence spectrum remains virtually the same. At first, we consider the effect of the ancillary halogen on the molecular orbital structure. To minimise the number of orbitals in consideration, we chose HOMO, HOMO-1, LUMO, and LUMO+1, being the most relevant orbitals to the two lowest singlet and triplet excitations (Fig. 7, Tables S4.3 and S4.5, ESI $\dagger$ ). Iodine as a stronger electron donor than chlorine affects mostly HOMO and HOMO-1, reducing the relative contribution of the $\pi$-conjugated $\mathrm{N}^{\wedge} \mathrm{C}^{\wedge} \mathrm{N}$ ligand to the molecular orbital. As a consequence, HOMO and HOMO-1 are localised mostly at the central atom and the ancillary iodide (Pt-I axis). Apart from that, the respective orbital contours in both 2 and $\mathbf{4}$ are nearly identical in each case. HOMO and HOMO-1 are visibly destabilised in 4 while LUMOs are stabilised, which leads to a narrower HOMO-LUMO gap in 4 than in 2 (Fig. S4.1 and S4.2, ESI $\dagger$ ). However, SOCdependent properties, such as zero-field splitting (ZFS) or the reciprocal triplet radiative rate $k_{\mathrm{r}}^{\mathrm{T}-1}$, are comparable, and the latter is in good agreement with experimental figures. Based on these results, we conclude that iodine per se does not introduce any significant SOC into the complex due to it being a heavier
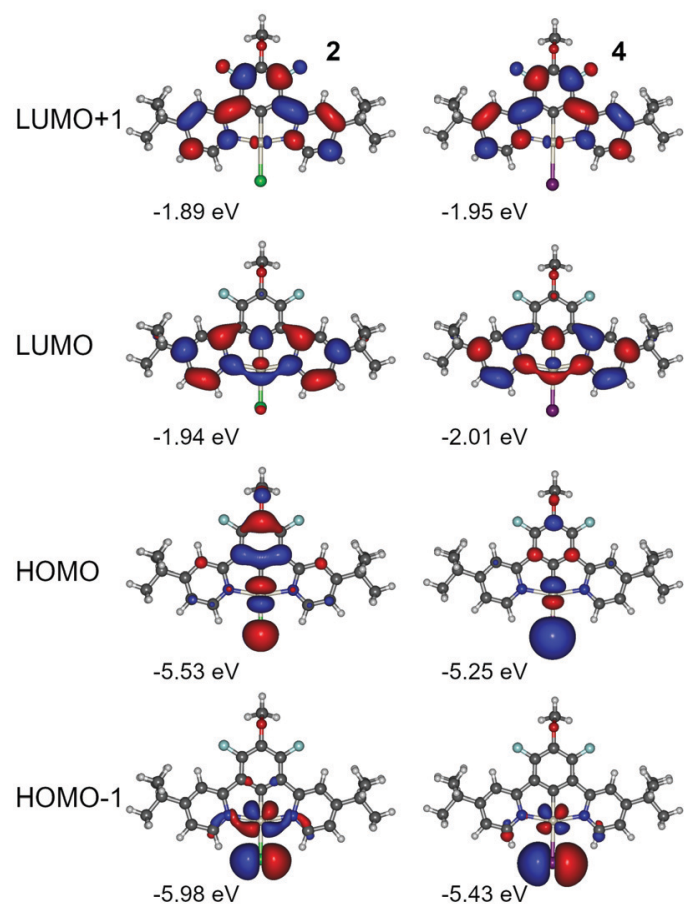

Fig. 7 Molecular orbital contour plots at the B3LYP/dev2-TZVP/ZORA level for monoplatinum(॥) complexes 2 and $\mathbf{4}$. atom than chlorine. It appears that the HOMO-LUMO overlap in 4 is smaller than in 2 leading to the $\Delta E_{\mathrm{ST}}$ in the former being nearly half the value of the latter (Table S4.1, ESI $\dagger$ ).

Having the above in mind, we now analyse the properties of 1 and 3. In this case we look into HOMO to HOMO-2 and LUMO as being most relevant to the lowest three singlet and triplet excitations (Fig. 8, Fig. S4.1, S4.2 and Tables S4.2 and S4.4, $\mathrm{ESI} \dagger$ ). We first note the orbital geometry is divergent between 1 and 3 with the HOMO in 3 being of similar geometry to the HOMO -2 in 1. At the same time HOMO-1 and HOMO-2 in 3 resemble HOMO and HOMO-1 in 1, respectively. The HOMOs in 3 exhibit a decreased contribution of the $\pi$-conjugated ligand to the molecular orbital than in complex 1 due to the presence of the ancillary iodide, which is similar to behaviour of complexes 2 and 4. The LUMO remains overall very similar in both dinuclear complexes and is located mostly on the electrondeficient pyrimidine linker. The HOMOs in complex 3 are significantly destabilised in respect to analogue $\mathbf{1}$ - consistently with behaviour of 2 and 4. Destabilisation of HOMO together with stabilisation of LUMO in 3 leads to a narrower energy gap than in the chloro analogue 1. The iodo-complex 3 shows a significantly smaller $\Delta E_{\mathrm{ST}}$ than its chloro analogue (Table S4.1, ESI $\dagger$ ). The ancillary iodide contributes to further reduction in HOMO-LUMO overlap, resulting in near orthogonality of the frontier molecular orbitals (MOs) and leading to reduction of $\mathrm{S}_{1}$ and $S_{n}$ energy without affecting the energy of the $T_{1}$ state. The frontier MO pattern of 3 resembles that presented by $\mathrm{B}-\mathrm{N}$ multiple resonance TADF emitters, ${ }^{46-48}$ yielding an excited state presenting a combination of small orbital overlap and negligible solvatochromic effects. We note that simulated ZFS is larger in $\mathbf{3}$ than in $\mathbf{1}$ and so is the $k_{\mathrm{r}}^{\mathrm{T}-1}$. According to the simulation (Table S4.1, ESI $\dagger$ ), ZFS in 3 is larger than in its monoplatinum(II) analogue 4 while the opposite can be said

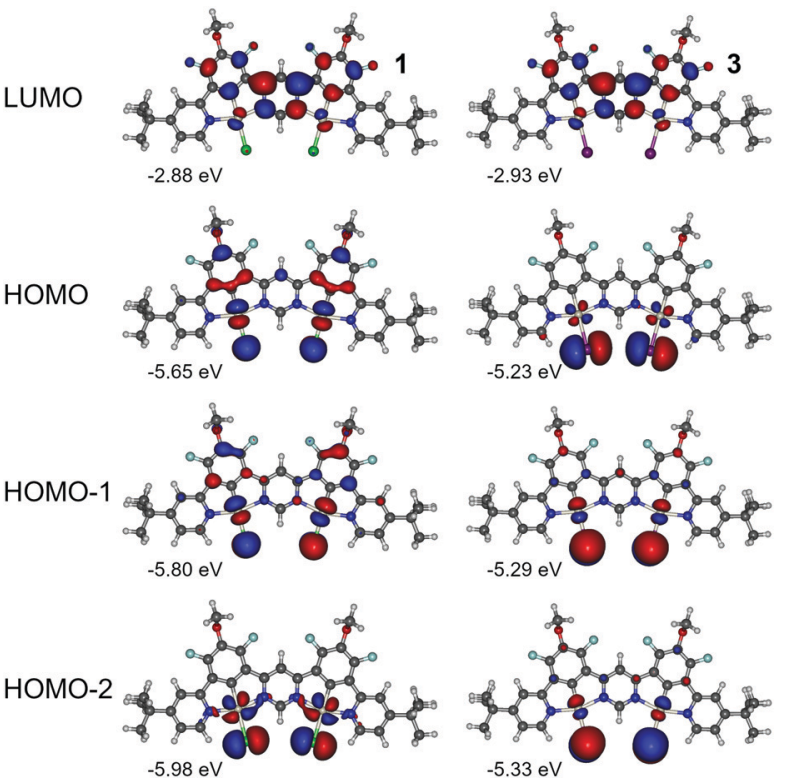

Fig. 8 Molecular orbital contour plots at the B3LYP/dev2-TZVP/ZORA level for diplatinum(II) complexes $\mathbf{1}$ and $\mathbf{3}$. 
about 1 and 2. So far, simulation appears to be consistent with the experimental data (Table 2).

In all three complexes, $1,2,4$, we find that both the $S_{1}$ and $T_{1}$ have a dominant HOMO $\rightarrow$ LUMO character while in 3 , the $\mathrm{T}_{1}$ is dominated by the HOMO-1 $\rightarrow$ LUMO transition involving $\mathrm{d}$ orbitals that are different from those of the $\mathrm{S}_{1}$ (HOMO $\rightarrow$ LUMO). Thus, the $\mathrm{S}_{1}-\mathrm{T}_{1}$ SOC matrix element (SOCME) in 3, $2276 \mathrm{~cm}^{-1}$, is at least 100 -fold larger than in the other three complexes. A large $\mathrm{S}_{1}-\mathrm{T}_{1} \mathrm{SOCME}$ and small $\Delta E_{\mathrm{ST}}$ should lead to a very high $k_{\mathrm{r}}^{\mathrm{T}}$. However, the oscillator strength $f\left(\mathrm{~T}_{1} \rightarrow \mathrm{S}_{0}\right)$ scales with that of the strongly coupled $S_{1}$ with an extremely small $f\left(\mathrm{~S}_{1} \rightarrow \mathrm{S}_{0}\right)=6 \times 10^{-5}$, thus this mixing does not provide a significant promotion of the radiative rate of $\mathrm{T}_{1} \cdot{ }^{49-51}$ The small $\mathrm{S}_{1} \rightarrow \mathrm{S}_{0}$ oscillator strength in 3 is a result of the near orthogonality between HOMO and LUMO in 3 . The value of $f\left(\mathrm{~S}_{1} \rightarrow \mathrm{S}_{0}\right)$ in 3 suggests a natural $\mathrm{S}_{1}$ decay lifetime of $\sim 100 \mu \mathrm{s}$ which is not only slower than the calculated natural phosphorescence decay rate of $\sim 60 \mu$ s but would render the molecule non-emissive through the $S_{1}$ due to incurring non-radiative processes. This contradiction demonstrates that a simple TDDFT calculation does not give a full picture of molecule 3 and SOC has to be taken into account.

SOC causes mixing between singlet and triplet states which often leads to equivocal spin character to the excited states in organometallic complexes. We identified that $\mathrm{S}_{1}$ contributes in $45 \%$ into triplet state $\Gamma_{3}$ which, however, retains small $f\left(\Gamma_{3} \rightarrow \Gamma_{0}\right)=1.6 \times 10^{-5}$ due to the low $f\left(\mathrm{~S}_{1} \rightarrow \mathrm{S}_{0}\right)$ as discussed in the previous paragraph. In this case, the lowest SOC-TDDFT state with singlet-like properties, such as large oscillator strength $f$, is $\Gamma_{4}$ which comprises $25 \% \mathrm{~S}_{2}, 59 \% \mathrm{~T}_{2}$, and $12 \%$ $\mathrm{T}_{3}$, among other states. $\Gamma_{4}$ is a singlet state with a strong contamination by triplet states, due to SOC. The oscillator strength $f\left(\Gamma_{4} \rightarrow \Gamma_{0}\right)=0.018$ gives $k_{\mathrm{r}}^{\mathrm{S}-1}=560 \mathrm{~ns}$, which is of the same order of magnitude as the experimentally derived value in polystyrene $k_{\mathrm{r}}^{\mathrm{S}-1}=100 \mathrm{~ns}$ (Table 2). Further to that, the $\Delta E_{\mathrm{ST}}$ calculated as $\Gamma_{1}-\Gamma_{4}=0.072 \mathrm{eV}$ is also close to the experimental figure $0.058 \mathrm{eV}$ in the same host. This demonstrates that the model used in this work correctly describes the excited state properties of complex 3 .

The theoretical picture of excited states in organometallic compounds, especially those with strong SOC, is consistent with a loss of unequivocal state multiplicity. ${ }^{52}$ For example, complex 1 undergoes radiative decay through an excited state with $\sim 70 \% \mathrm{~S}_{1}$ character, whereas in 3 it is only $25 \% \mathrm{~S}_{2}$. In this case the luminescence arising from the energetically upper state, attributed to the $S_{1}$ in metal-free systems, cannot always be identified as conventional fluorescence. However, due to the similar experimental picture of organometallic emitters to classical TADF molecules, we consider the term to be also applicable to the former and thus the unequivocal attribution of state multiplicity, i.e. $\mathrm{S}_{1}$ and $\mathrm{T}_{1}$, to still be helpful in the description of experimental results. In this case, the emissive states with substantially larger transition oscillator strength $f\left(\Gamma_{n} \rightarrow \Gamma_{0}\right)$ than the lowest emissive triplet states $\Gamma_{1-3}$ and clear admixture of singlets can be treated as singlets despite being strongly contaminated by triplet state admixtures.

The conclusions presented in this section strengthen the view that the profound luminescence properties of many dinuclear platinum(II) complexes do not necessarily come from the increased metal contribution to the excited state. Instead, it appears that the two metals benefit the complex by provision of additional singlet states involving metal d orbitals that are different from those of $\mathrm{T}_{1}$, while also bringing them closer together. $^{8,9,42}$ The ancillary iodide acts in a similar way in complex 3, by destabilising HOMOs and reducing energy difference between HOMO and HOMO-1 etc. In extreme situations, this also leads to re-ordering of orbital patterns, which promotes even stronger SOC.

\section{OLED devices}

Complex 3 shows a lesser tendency to form NIR-emitting excimers than its chloro analogue 1. It is therefore more amenable to application in an OLED, taking advantage of its efficient monomolecular luminescence through TADF. The complex is highly soluble in toluene, in contrast to $\mathbf{1}$ which only shows negligible solubility in this solvent. This has allowed more complex OLED structures to be explored, incorporating high molecular weight poly( $N$-vinylcarbazole) $(\mathrm{PVKH})$ as the hole transport and electron blocking layer (Devices 1 and 2 in Table 3). The device structures used are shown in the ESI $\dagger$ (Table S7.1). The electroluminescence spectrum of Devices 1 and 2 with $5 \%$ emitter doping is dominated by the red (CIE $0.62,0.36)$ monomolecular emission of $3\left(\lambda_{\mathrm{EL}}=612 \mathrm{~nm}\right)$ with a shoulder of a NIR-emitting excimer at 800-900 nm (Fig. 9). The luminescence at $\lambda_{\mathrm{EL}}=612 \mathrm{~nm}$ shows a more narrowband $(\mathrm{FWHM}=89 \mathrm{~nm})$ distribution than that of typical chargetransfer red TADF emitters, ${ }^{16}$ thanks to the multiple resonance-like orbital pattern in the complex 3. The shoulder at $450-550 \mathrm{~nm}$ in the electroluminescence spectrum of Device 1 is attributed to the exciplex formed at the interface between PVKH and PO-T2T ${ }^{53}$ \{2,4,6-tris[3-(diphenylphosphinyl)phenyl]-1,3,5-triazine $\}$ from the mCP:PO-T2 $\mathrm{T}^{54}$ host $\{\mathrm{mCP}-1,3-$ bis(carbazol-9-yl)benzene $\}$. Device 2, which uses a different electron transport material in the blend namely PBD

Table 3 Characteristics of OLED devices fabricated with 3 as an emitter. Device structures are shown in the ESI (Table S7.1)

\begin{tabular}{|c|c|c|c|c|c|c|c|}
\hline Device & $x,^{a} \%$ & $\lambda_{\mathrm{el}},{ }^{b} \mathrm{~nm}$ & $\% \lambda>700,{ }^{c} \mathrm{~nm}$ & $\Phi_{\mathrm{PL}}^{d}$ & $\mathrm{EQE}_{\max },{ }^{e} \%$ & Max. luminance, $\mathrm{cd} \mathrm{m}^{-2}$ & Max. radiosity, $\mathrm{mW} \mathrm{cm}^{-2}$ \\
\hline Dev 2 & 5 & 612 & 21 & $0.11 \pm 0.05$ & 2.29 & 1840 & 2.86 \\
\hline Dev 3 & 33 & 782 & 69 & $0.04 \pm 0.02$ & 0.36 & 160 & 0.82 \\
\hline
\end{tabular}

${ }^{a}$ Weight doping concentration of 3 in the emissive layer. ${ }^{b}$ Electroluminescence maxima. ${ }^{c}$ Percent of spectral power at wavelengths above $700 \mathrm{~nm}$. ${ }^{d}$ Photoluminescence quantum yield of the emissive layer in nitrogen. ${ }^{e}$ Device maximum external quantum efficiency. 

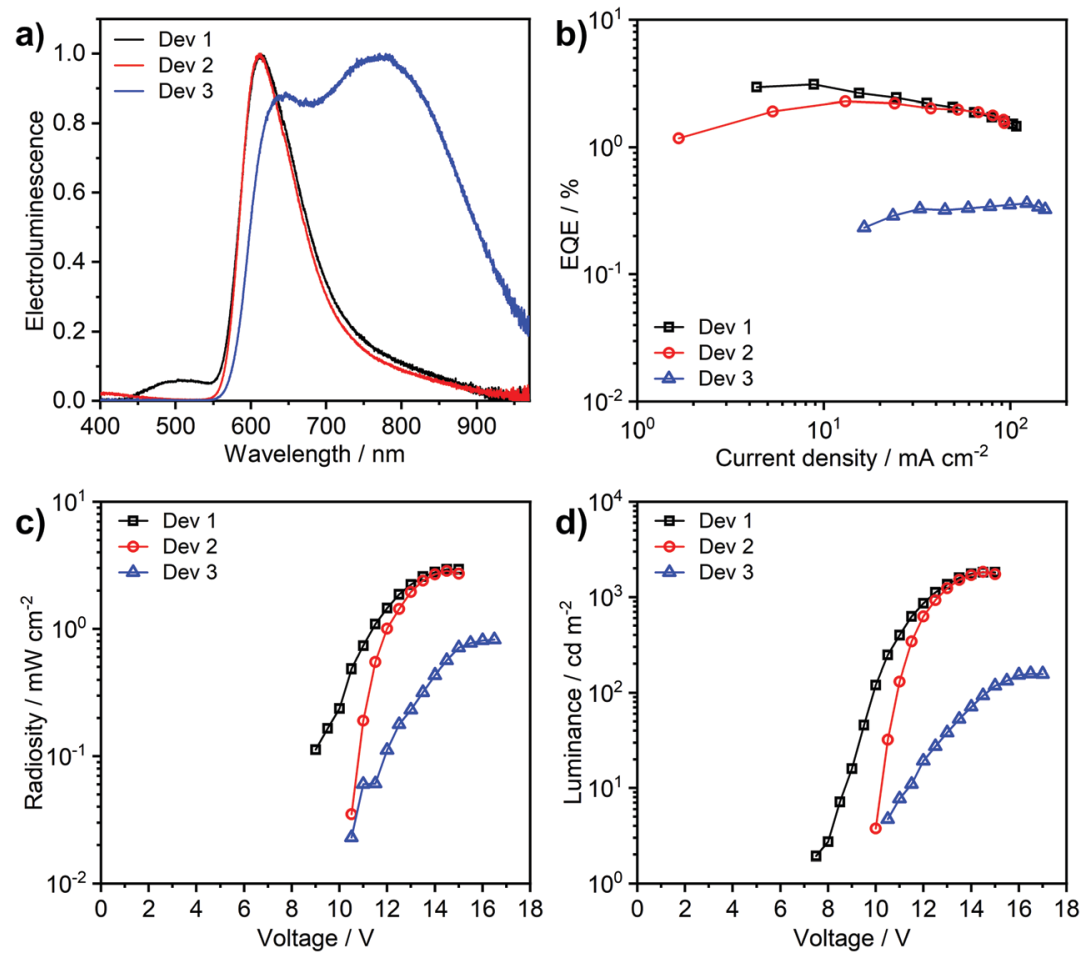

Fig. 9 Characteristics of devices 1-3: (a) electroluminescence spectra; (b) external quantum efficiency (EQE) vs. current density; (c) radiosity-voltage characteristics; (d) luminance-voltage characteristics.

\{2-(4-biphenyl)-5-(4-tert-butylphenyl)-1,3,4-oxadiazole\}, does not show any electroluminescence in this region. The OLED produced with higher, 33\% emitter load (Device 3) demonstrates strong contribution from an NIR excimer $\left(\lambda_{\mathrm{EL}}=782 \mathrm{~nm}\right)$, although the higher energy electroluminescence band at $\lambda_{\mathrm{EL}}=612 \mathrm{~nm}$ does remain significant. We have also fabricated a non-doped device with the complex used neat as the emitting layer (Fig. S7.2, ESI $\dagger$ ), but the electroluminescence was too weak to be fully characterised. This OLED shows excimer electroluminescence peaking at $824 \mathrm{~nm}$, a longer wavelength than the value of $805 \mathrm{~nm}$ reported for the chloro analogue 1. Interestingly, a small contribution from the monomolecular emission can still be noticed in the electroluminescence spectrum of the non-doped device.

The device external quantum efficiency agrees with the $\Phi_{\mathrm{PL}}$ in film in each case (Table 3 ). We note that the long-wavelength excimer formed from 3 has very low emission efficiency and acts as a luminescence quencher in solid film, significantly reducing the $\Phi_{\mathrm{PL}}$ from the high yields reported in solution. Therefore, there is still a need for further improvement of complexes such as 3 towards reducing intermolecular interactions in solid film, if highly-efficient multiple resonace-like TADF OLEDs are to be achieved.

\section{Conclusions}

We report a relatively simple practical strategy for enhancing the TADF properties of diplatinum(II) complexes via substitution of the ancillary ligand. The newly reported, delayed fluorescence di-Pt(II) complex 3 shows a very small $E_{\mathrm{a}}=58 \pm$
$3 \mathrm{meV}$ in polystyrene and a large radiative decay rate in toluene, $k_{\mathrm{r}}=4.3 \times 10^{5} \mathrm{~s}^{-1}$, comparable with those of state-of-the-art iridium(III) complexes. ${ }^{55}$ Thanks to the TADF mechanism, a simple substitution of the ancillary ligand with iodide results in a radiative decay rate $\sim 3$-fold larger than in the chloro analogue 1. We identify that, although the ancillary iodide does improve luminescent characteristics of complex 3 thanks to the TADF mechanism, it also leads to somehow greater SOC in the dinuclear complex. However, the role of iodine does not relate to its intrinsic SOC constant, but rather to its role in destabilising the highest occupied molecular orbitals in the molecule. Such a situation leads to a better mixing of singlet character into the triplet states as there is a large number of $S_{n}$ states energetically close to the $T_{1}$ in complex 3 .

To demonstrate potential applicability of this group of complexes in OLEDs, we have produced solution-processed devices demonstrating relatively narrow-band red electroluminescence $\left(\lambda_{\mathrm{EL}}=612 \mathrm{~nm}, \mathrm{FWHM}=89 \mathrm{~nm}\right)$, with EQE of $3.11 \%$. Some improvements are clearly necessary in order to prevent the occurrence of luminescence-quenching through aggregation and/or excimer formation in the solid state, in order to achieve highly efficient OLEDs.

\section{Author contributions}

P. P. - conceptualization, formal analysis, investigation, visualization, writing - original draft, writing - review and editing; A. V. Z. investigation; A. S. - investigation; J. A. G. W. - conceptualization, funding acquisition, project administration, supervision, 
writing - review and editing; V. N. K. - conceptualization, funding acquisition, project administration, writing - original draft, writing review and editing; F. B. D. - funding acquisition, project administration, resources, validation, writing - original draft, writing - review and editing.

\section{Conflicts of interest}

There are no conflicts to declare.

\section{Acknowledgements}

We thank EPSRC (grant refs EP/S012788/1 and EP/S01280X) for support of this work. We are grateful to Dr Dmitry Yufit at Durham Chemistry for determining the crystal structure of complex 3 and for his continued assistance with crystallography. The authors thank Dr Gleb Baryshnikov for fruitful discussions. This work made use of the facilities of the Hamilton HPC Service of Durham University.

\section{Notes and references}

1 H. Yersin, A. F. Rausch, R. Czerwieniec, T. Hofbeck and T. Fischer, Coord. Chem. Rev., 2011, 255, 2622-2652.

2 H. Yersin, Highly Efficient OLEDs with Phosphorescent Materials, Wiley, 2008.

3 C. Murawski, K. Leo and M. C. Gather, Adv. Mater., 2013, 25, 6801-6827.

4 Y. J. Cho, K. S. Yook and J. Y. Lee, Adv. Mater., 2014, 26, 4050-4055.

5 B. Geffroy, P. le Roy and C. Prat, Polym. Int., 2006, 55, 572-582.

6 M. J. Leitl, V. A. Krylova, P. I. Djurovich, M. E. Thompson and H. Yersin, J. Am. Chem. Soc., 2014, 136, 16032-16038.

7 A. Bossi, A. F. Rausch, M. J. Leitl, R. Czerwieniec, M. T. Whited, P. I. Djurovich, H. Yersin and M. E. Thompson, Inorg. Chem., 2013, 52, 12403-12415.

8 M. Z. Shafikov, P. Pander, A. V. Zaytsev, R. Daniels, R. Martinscroft, F. B. Dias, J. A. G. Williams and V. N. Kozhevnikov, J. Mater. Chem. C, 2021, 9, 127-135.

9 M. Z. Shafikov, R. Daniels, P. Pander, F. B. Dias, J. A. G. Williams and V. N. Kozhevnikov, ACS Appl. Mater. Interfaces, 2019, 11, 8182-8193.

10 R. E. Daniels, S. Culham, M. Hunter, M. C. Durrant, M. R. Probert, W. Clegg, J. A. G. Williams and V. N. Kozhevnikov, Dalton Trans., 2016, 45, 6949-6962.

11 Z. Q. Zhu, C. Do Park, K. Klimes and J. Li, Adv. Opt. Mater., 2019, 7, 1801518.

12 T. Hofbeck, U. Monkowius and H. Yersin, J. Am. Chem. Soc., 2015, 137, 399-404.

13 D. N. Kozhevnikov, V. N. Kozhevnikov, M. Z. Shafikov, A. M. Prokhorov, D. W. Bruce and J. A. G. Williams, Inorg. Chem., 2011, 50, 3804-3815.

14 A. Zampetti, A. Minotto and F. Cacialli, Adv. Funct. Mater., 2019, 29, 1807623.
15 H. Uoyama, K. Goushi, K. Shizu, H. Nomura and C. Adachi, Nature, 2012, 492, 234-238.

16 Y. Tao, K. Yuan, T. Chen, P. Xu, H. Li, R. Chen, C. Zheng, L. Zhang and W. Huang, Adv. Mater., 2014, 26, 7931-7958.

17 A. S. Romanov, L. Yang, S. T. E. Jones, D. Di, O. J. Morley, B. H. Drummond, A. P. M. Reponen, M. Linnolahti, D. Credgington and M. Bochmann, Chem. Mater., 2019, 31, 3613-3623.

18 R. Czerwieniec, J. Yu and H. Yersin, Inorg. Chem., 2011, 50, 8293-8301.

19 R. Czerwieniec and H. Yersin, Inorg. Chem., 2015, 54, 4322-4327.

20 A. S. Romanov, S. T. E. Jones, L. Yang, P. J. Conaghan, D. Di, M. Linnolahti, D. Credgington and M. Bochmann, Adv. Opt. Mater., 2018, 6, 1801347.

21 D. Di, A. S. Romanov, L. Yang, J. M. Richter, J. P. H. Rivett, S. Jones, T. H. Thomas, M. A. Jalebi, R. H. Friend, M. Linnolahti, M. Bochmann and D. Credgington, Science, 2017, 356, 159-163.

22 G. Li, Q. Chen, J. Zheng, Q. Wang, F. Zhan, W. Lou, Y. F. Yang and Y. She, Inorg. Chem., 2019, 58, 14349-14360.

23 P. W. Zach, S. A. Freunberger, I. Klimant and S. M. Borisov, ACS Appl. Mater. Interfaces, 2017, 9, 38008-38023.

24 K. Chan, T. Lam, D. Yu, L. Du, D. L. Phillips, C. Kwong, G. S. M. Tong, G. Cheng and C. Che, Angew. Chem., 2019, 131, 15038-15042.

25 A. Endo, M. Ogasawara, A. Takahashi, D. Yokoyama, Y. Kato and C. Adachi, Adv. Mater., 2009, 21, 4802-4806.

26 P. Pander, R. Daniels, A. V. Zaytsev, A. Horn, A. Sil, T. J. Penfold, J. A. G. Williams, V. N. Kozhevnikov and F. B. Dias, Chem. Sci., 2021, 12, 6172-6180.

27 M. Z. Shafikov, R. Martinscroft, C. Hodgson, A. Hayer, A. Auch and V. N. Kozhevnikov, Inorg. Chem., 2021, 60, 1780-1789.

28 P. Pander, A. V. Zaytsev, A. Sil, J. A. G. Williams, P.-H. Lanoe, V. N. Kozhevnikov and F. B. Dias, J. Mater. Chem. C, 2021, 9, 10276-10287.

29 J. A. G. Williams, Chem. Soc. Rev., 2009, 38, 1783.

30 A. F. Rausch, L. Murphy, J. A. G. Williams and H. Yersin, Inorg. Chem., 2012, 51, 312-319.

31 P.-H. Lanoë, C. M. Tong, R. W. Harrington, M. R. Probert, W. Clegg, J. A. G. Williams and V. N. Kozhevnikov, Chem. Commun., 2014, 50, 6831-6834.

32 C. A. Parker and C. G. Hatchard, Trans. Faraday Soc, 1961, 57, 1894. 33 C. Würth, M. Grabolle, J. Pauli, M. Spieles and U. ReschGenger, Nat. Protoc., 2013, 8, 1535-1550.

34 The IUPAC Compendium of Chemical Terminology, ed. V. Gold, International Union of Pure and Applied Chemistry (IUPAC), Research Triangle Park, NC, 2019.

35 J. R. Kirchhoff, R. E. Gamache, M. W. Blaskie, A. A. Del Paggio, R. K. Lengel and D. R. McMillin, Inorg. Chem., 1983, 22, 2380-2384.

36 Y. Liu, G. Zhan, Z.-W. Liu, Z.-Q. Bian and C.-H. Huang, Chinese Chem. Lett., 2016, 27, 1231-1240.

$37 \mathrm{~J} . \mathrm{Xu}, \mathrm{A}$. Takai, Y. Kobayashi and M. Takeuchi, Chem. Commun., 2013, 49, 8447.

38 H. Chen, L. Xu, X. Ma and H. Tian, Polym. Chem., 2016, 7, 3989-3992. 
39 J. Wang, X. Gu, H. Ma, Q. Peng, X. Huang, X. Zheng, S. H. P. Sung, G. Shan, J. W. Y. Lam, Z. Shuai and B. Z. Tang, Nat. Commun., 2018, 9, 2963.

40 Z. Y. Zhang, Y. Chen and Y. Liu, Angew. Chem., Int. Ed., 2019, 58, 6028-6032.

$41 \mathrm{M}$. Shimizu, in Principles and Applications of AggregationInduced Emission, ed. Y. Tang and B. Z. Tang, Springer International Publishing, Cham, 2019, pp. 43-76.

42 M. Z. Shafikov, A. V. Zaytsev and V. N. Kozhevnikov, Inorg. Chem., 2021, 60, 642-650.

43 B. D. Rossenaar, D. J. Stufkens and A. Vlček, Inorg. Chem., 1996, 35, 2902-2909.

44 A. Cannizzo, A. M. Blanco-Rodríguez, A. El Nahhas, J. Šebera, S. Záliš, A. Vlček and M. Chergui, J. Am. Chem. Soc., 2008, 130, 8967-8974.

45 J. M. Younker and K. D. Dobbs, J. Phys. Chem. C, 2013, 117, 25714-25723.

46 T. Hatakeyama, K. Shiren, K. Nakajima, S. Nomura, S. Nakatsuka, K. Kinoshita, J. Ni, Y. Ono and T. Ikuta, Adv. Mater., 2016, 28, 2777-2781.
47 J. M. Ha, S. H. Hur, A. Pathak, J.-E. Jeong and H. Y. Woo, NPG Asia Mater., 2021, 13, 53.

48 S. M. Suresh, E. Duda, D. Hall, Z. Yao, S. Bagnich, A. M. Z. Slawin, H. Bässler, D. Beljonne, M. Buck, Y. Olivier, A. Köhler and E. Zysman-Colman, J. Am. Chem. Soc., 2020, 142, 6588-6599.

49 G. Baryshnikov, B. Minaev and H. Ågren, Chem. Rev., 2017, 117, 6500-6537.

50 K. Nozaki, J. Chinese Chem. Soc., 2006, 53, 101-112.

51 K. Mori, T. P. M. Goumans, E. van Lenthe and F. Wang, Phys. Chem. Chem. Phys., 2014, 16, 14523-14530.

52 G. A. Crosby, K. W. Hipps and W. H. Elfring, J. Am. Chem. Soc., 1974, 96, 629-630.

53 P. Pander, S. Gogoc, M. Colella, P. Data and F. B. Dias, ACS Appl. Mater. Interfaces, 2018, 10, 28796-28802.

54 M. T. Walden, P. Pander, D. S. Yufit, F. B. Dias and J. A. G. Williams, J. Mater. Chem. C, 2019, 7, 6592-6606.

55 T. Sajoto, P. I. Djurovich, A. B. Tamayo, J. Oxgaard, W. A. Goddard and M. E. Thompson, J. Am. Chem. Soc., 2009, 131, 9813-9822. 